\title{
Sialoendoscopia: una nueva alternativa en el tratamiento de la patología salival. Nuestra experiencia
}

Peter M. Baptista ${ }^{a}$, Carlos Gimeno-Vilar ${ }^{a}$, Jorge A. Rey-Martínez $z^{a}$ y Manuele Casale-Falcone ${ }^{b}$

a Departamento de Otorrinolaringología. Clínica Universitaria de Navarra. Pamplona. Navarra. España.

bepartamento de Otorrinolaringología. Universidad Campus Biomédico. Roma. Italia.

Objetivos: La sialoendoscopia es un procedimiento cuya finalidad es visualizar los conductos salivales y sus procesos patológicos. Se utiliza como método diagnóstico, para descartar procesos inflamatorios de las glándulas (sialoendoscopia diagnóstica) y para tratar áreas patológicas (estenosis, retirar cuerpos extraños o cálculos), mediante instrumental (sialoendoscopia intervencionista). Queremos mostrar la disminución de extirpación glandular.

Pacientes y método: Se estudia a 8 pacientes intervenidos con sialoendoscopia.

Resultados: En el 50\% se trataba de sialolitiasis y en el otro $50 \%$, de sialodenitis crónica. En las sialolitiasis la sialoendoscopia intervencionista permitió extraer el cálculo en 2 (50\%) casos. En el resto la sialoendoscopia permitió confirmar el diagnóstico en todos los casos.

Conclusiones: La sialoendoscopia es un nueva técnica que nos permite diagnosticar, tratar y hacer un adecuado seguimiento de las sialodenitis, sialolitiasis y otras afecciones glandulares.

Palabras clave: Sialoendoscopia. Sialolitiasis. Sialodenitis.
Sialoendoscopy: A New Alternative for the Treatment of Salivary Pathology. Our Experience

Objectives: Sialoendoscopy is a procedure used to visualize the salivary ducts and their pathology. It can be used either as a diagnostic method to rule out inflammatory processes in the parotid and submandibular glands (diagnostic sialoendoscopy) or to treat pathological areas (stenosis, extract foreign bodies or sialolithiasis) through the use of appropriate instruments (interventionist sialoendoscopy). We attempt to prove a declining rate of salivary gland excision. Patients and method: Sialoendoscopy was performed in 8 patients.

Results: Of these, $50 \%$ of patients were diagnosed as having sialolithiasis and the other $50 \%$ had chronic sialoadenitis. In patients with sialolithiasis, sialoendoscopy allowed the extraction of the calculus in two patients (50\%). In the remainder, sialoendoscopy provided confirmation of the diagnosis in all cases.

Conclusions: Sialoendoscopy is a new technique for use in the diagnosis, treatment and post-operative management of sialolithiasis, sialoadenitis and other salivary gland pathologies.

Key words: Sialoendoscopy. Sialolithiasis. Sialoadenitis.

\section{INTRODUCCIÓN}

La sialoendoscopia se realizó por primera vez a principios de los años noventa. Modificaciones hechas a endoscopios rígidos llevaron a Oded Nahlieli y a Francis Marchal a confeccionar endoscopios semirrígidos de diseño similar, pero con características discretamente diferentes ${ }^{1,2}$.

Los autores no manifiestan ningún conflicto de intereses.

Correspondencia: Dr. C. Gimeno Vilar.

Avda. Pío XII, 36. 31620 Pamplona. Navarra. España.

Correo electrónico: cgimeno@unav.es

Recibido el 18-10-2007.

Aceptado para su publicación el 21-1-2008.
A partir de éstos se ha ido desarrollando una nueva técnica actualmente en expansión. Las observaciones de Nahlieli y Marchal han sido muy importantes para establecer unos criterios diagnósticos en la sialoendoscopia.

La sialoendoscopia se puede utilizar como método diagnóstico, para descartar causas inflamatorias de las glándulas, para tratar áreas patológicas (estenosis o cálculos) mediante instrumental. Las indicaciones son todas las inflamaciones de las glándulas salivales de origen desconocido $^{3,5,9}$.

A pesar de su simplicidad, la sialoendoscopia es un procedimiento con retos, especialmente la intervencionista. La utilización del sialoendoscopio rígido requiere de una experiencia extensa por el potencial riesgo de perforación del conducto salival, con lesión de estructuras vasculares y 
neurales vecinas. El endoscopio sólo debe ser utilizado bajo visión adecuada. Los cálculos distales o la estrechez del conducto pueden limitar de una forma importante el avance del endoscopio y su utilidad.

La sialolitiasis afecta al 1,2\% de las glándulas salivales. De acuerdo con datos publicados, los cálculos salivales se encuentran en un 80-90\% de los casos en la glándula submaxilar ${ }^{5}$. De forma y composición variable tienen una tasa de crecimiento de $1 \mathrm{~mm}$ por año. Pueden flotar o encontrarse parcialmente adheridos a las paredes del conducto. La sialolitiasis puede ser transitoria o complicarse con una infección bacteriana ${ }^{4,5}$.

Los tapones de moco se encuentran compuestos por células descamativas epiteliales e inflamatorias. Se consideran cambios degenerativos de las células epiteliales del conducto, por lo que pueden desempeñar un papel determinante como factor de obstrucción y contribuir a la estrechez de la zona y la formación de pólipos y/o cálculos.

La permeabilidad del conducto salival se puede alterar por una estenosis, que causa síntomas clínicos parecidos a una litiasis. Se localiza endoscópicamente la estrechez, se visualiza y, mediante un sistema de dilatadores rígidos o dilatadores de balón, se resuelve $e^{3,5}$.

Es nuestro objetivo comprobar la utilidad y la eficacia de la sialoendoscopia para evitar intervenciones con mayor morbilidad (exéresis glandular).

\section{MÉTODOS}

\section{Descripción de la técnica}

Es realizada por un cirujano principal y un ayudante. El instrumental se compone de: sondas de diámetros crecientes (745847-56; Karl Storz Endoscope, Alemania), pinzas de disección, tijera angulada, pinzas de biopsia, sialoendosco- pio 11575 de Marchall (Karl Storz, diámetro externo de $1,3 \mathrm{~mm}$ ) de dos canales de $0,25 \mathrm{~mm}$ y de $0,65 \mathrm{~mm}$, fuente de luz y cable, abridor de boca y retenedor de 1 labio ${ }^{5}$ (fig. 1).

Hay 4 abordajes para la introducción del endoscopio: a través del orificio natural, mediante papilotomía posterior al orificio natural del conducto, realizando la búsqueda del conducto en una zona más posterior o a través de su abertura $^{5}$.

Infiltramos a nivel de la papila lidocaína al 10\%, no directamente sobre ésta para evitar perder el orificio y lograr una adecuada visualización. Introducimos los dilatadores salivales desde la sonda más fina (0000). Para introducir la sonda, se tracciona la papila de la glándula. Esta tracción permite rectificar la tortuosidad de los conductos. Seguidamente se introduce la sonda 000 , seguida por la 00 . Cada dos números se aplica el dilatador. Luego se introduce la sonda $0 \mathrm{y}$, posteriormente, las sondas hasta la número 6. Se debe evitar forzar, y cuando no se pueda pasar a un número superior, retroceder a una sonda más fina. En caso de que haya una estrechez importante sobre la salida del conducto de Wharton podemos realizar una pequeña papilotomía en los primeros $5 \mathrm{~mm}$. Se debe evitar una papilotomía del conducto de Stenon. Una vez lograda una dilatación lo suficientemente amplia, se infiltrará solución anestésica, lidocaína al 1\% con adrenalina (1:100.000). Se introduce el endoscopio aproximadamente $1 \mathrm{~cm}$ y se lava el conducto con suero o anestesia hasta lograr una visión clara.

Una maniobra recomendable es seguir el punto negro en la pantalla del monitor que es compatible con el lumen del conducto (fig. 2).

Con las sialolitiasis utilizamos sondas láser de holmio adaptadas al fibroscopio (fibras de $365 \mu \mathrm{m}$; ref. 27750172) que fragmentan los cálculos con visión directa, y se recogen con una cesta ${ }^{6}$ (fig. 3).

Figura 1. A: instrumental quirúrgico. B: sialoendoscopio. C: abordaje de la glándula submaxilar.

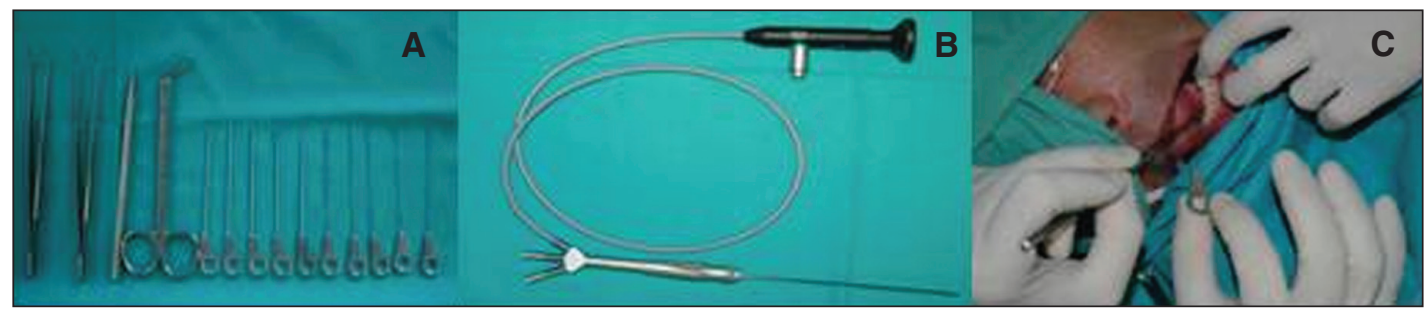

Figura 2. Imágenes de endoscopia. A: conducto de Stenon, se observa la vascularización de las paredes y su aspecto anillado. B: conducto de Wharton. El endoscopio llega hasta las ramificaciones (flecha). C: lumen del conducto, punto negro que debemos seguir en la pantalla al introducir el endoscopio.
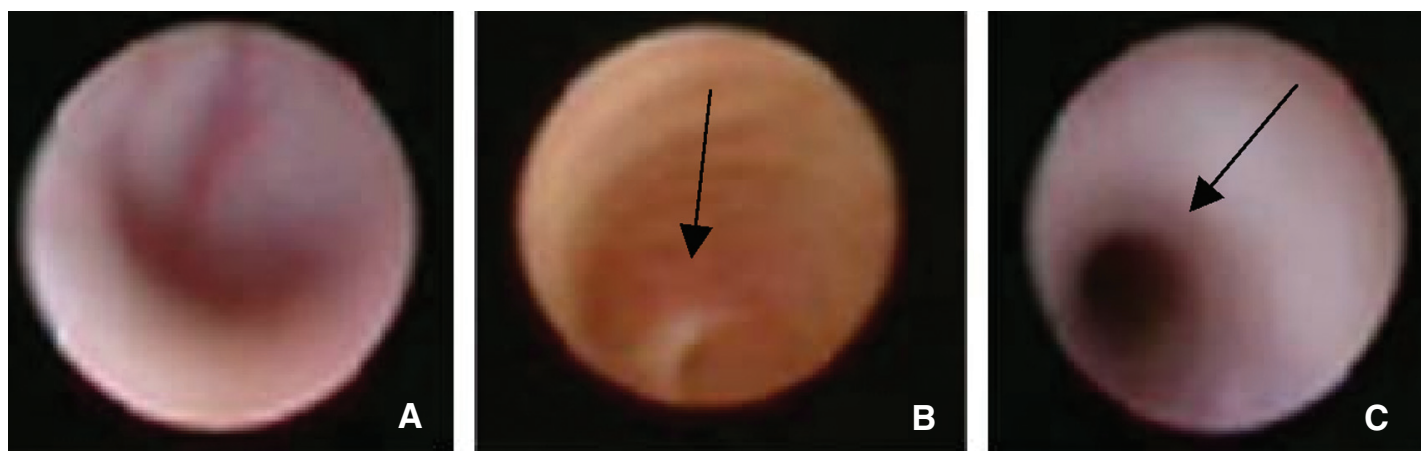


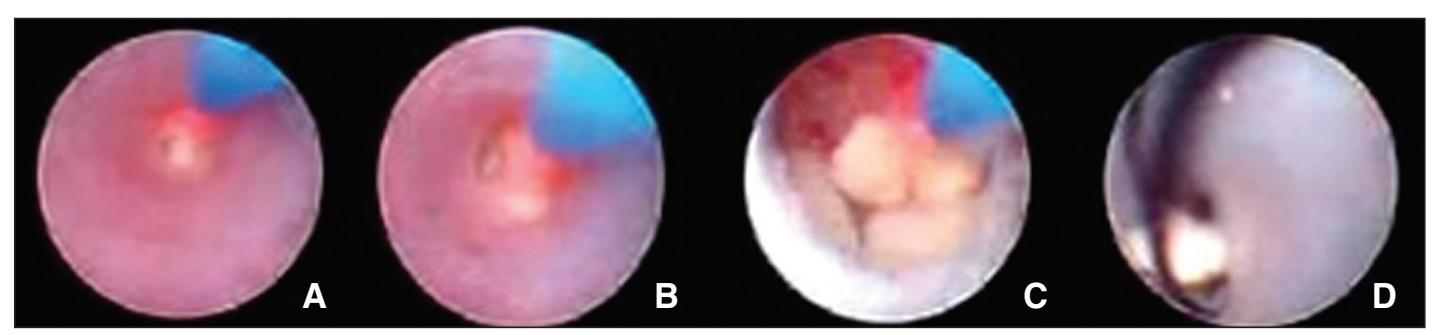

Figura 3. Se aprecia que el cálculo que obstruye la luz del conducto es fragmentado mediante láser para facilitar su extracción y los restos son recogidos mediante una cesta.
Mantenemos profilaxis antibiótica las primeras $48 \mathrm{~h}$, y se realizan controles clínicos después de cada intervención. No es preciso un ingreso mayor que $24 \mathrm{~h}$. Sólo se debe vigilar a los pacientes con rotura del conducto de Wharton o marsupialización extensa ante el riesgo de difusión del edema y/o infección del suelo de la boca que puede llegar a afectar a la vía aérea.

Durante el período diciembre 2005 a septiembre 2007 hemos realizado sialoendoscopia en 8 pacientes con síntomas de obstrucción o inflamación crónica de glándulas salivales mayores. De los 8 pacientes, 6 eran mujeres y 2, varones, con una media de edad de 38 años. En 6 casos fueron glándulas submaxilares y en 2, parótidas. De éstas, 4 fueron diagnosticadas como obstrucción y 4 como sialodenitis crónica (tabla I).

Se realizó preoperatoriamente un sialorresonancia magnética. En todos los casos se realizó con anestesia general, con una duración media de $120 \mathrm{~min}$. En los casos con litiasis utilizamos sondas láser de holmio para fragmentar los cálculos.

En los casos con sialodenitis crónica, después de explorar el conducto, se observó los conductos principales y secundarios con estrecheces y adherencias algodonosas. En éstos se lavó el conducto con suero y lidocaína al 1\% y se atravesó con la cesta a modo de dilatador.

\section{RESULTADOS}

Obtuvimos un éxito en la extracción de litiasis (4 pacientes) del $50 \%$, realizamos 2 exéresis glandulares debido al gran tamaño de los cálculos (12 y $15 \mathrm{~mm}$ ), no se consiguió fragmentar lo suficiente el cálculo con el láser (pacientes 3 y
7). En los 4 casos diagnosticados de sialodenitis crónica obtuvimos un éxito del $75 \%$. En uno de los casos (paciente 8) la sintomatología mejoró durante las 3 semanas tras la cirugía, pero reapareció, y se realizó una parotidectomía superficial ligando el conducto, con lo que los síntomas remitieron.

Tuvimos un $25 \%$ de complicaciones, con dos edemas linguales. No se pudo desentubar inmediatamente a los pacientes que ingresaron en la UCI hasta la resolución del edema ( $6 \mathrm{~h}$ tras la intervención). A un paciente se le dio el alta al día siguiente, y el otro permaneció ingresado 2 días más debido a la exéresis glandular, por el gran tamaño del cálculo sufrió una ránula traumática que se resolvió a los 2 días (tratamiento antibiótico y antiinflamatorio) (tabla I).

La revisión postoperatoria se realizó al mes de la intervención, con revisiones a los 3 meses y al año. Con un seguimiento de 1 año y 9 meses en el primer caso.

\section{DISCUSIÓN}

La sialoendoscopia puede realizarse ambulatoriamente ${ }^{5}$ o con anestesia general. En nuestro centro se realiza con anestesia general para facilitar la maniobrabilidad del cirujano y evitar molestias innecesarias al paciente.

Las vías de abordaje a través del orificio natural o mediante una papilotomía posterior conllevan menos complicaciones, favorecen una correcta entrada en la luz ductal y evitan la formación de falsas vías. Al introducir el endoscopio irrigamos a la vez que progresamos buscando dilatar el conducto, ya que en condiciones basales la luz es virtual y sólo de este modo logramos observarla adecuadamente. Las maniobras deben ser lo menos traumáticas posibles para evitar perforaciones y estenosis tardía.

Tabla I. Descripción de la muestra

\begin{tabular}{|c|c|c|c|c|c|c|c|}
\hline Paciente & Edad & Sexo & Dx-pre & $D x$-post & Prueba diagnóstica & Glándula afectada & Complicación \\
\hline 1 & 35 & Mujer & Adenitis & Estrechez & SialoRM & Submaxilar derecha & No \\
\hline 2 & 42 & Mujer & Litiasis & Litiasis & SialoRM & Submaxilar derecha & No \\
\hline 4 & 22 & Mujer & Litiasis & Litiasis & SialoRM & Submaxilar izquierda & Edema lingual \\
\hline 5 & 20 & Mujer & Litiasis & Estrechez & SialoRM & Submaxilar derecha & No \\
\hline 7 & 38 & Varón & Litiasis & Litiasis & SialoRM & Submaxilar izquierda & Edema lingual/ránula \\
\hline 8 & 58 & Mujer & Adenitis & Estrechez & SialoRM & Parótida derecha & No \\
\hline
\end{tabular}

Dx-pre: diagnóstico antes de la intervención; Dx-post: diagnóstico después de la intervención; sialoRM: sialorresonancia magnética. 
Con la sialoendoscopia podemos explorar el epitelio de revestimiento de los conductos, lo que nos permite encontrar cálculos que no pueden ser diagnosticados por otros métodos de exploración (radiografía, tomografía computarizada o ecografía). Así los beneficios diagnósticos y terapéuticos de la sialoendoscopia son mayores que los métodos convencionales de radiología ${ }^{6}$, a pesar de ello muchos autores recomiendan el uso de la sialografía. Además, se ha logrado evitar la extirpación innecesaria de glándulas salivales y se ha descrito un caso de carcinoma mucoepidermoide de conducto salival. Por lo que en un futuro podremos diagnosticar tumores con más frecuencia y en estadios más tempranos.

Según la experiencia de algunos autores, la glándula submaxilar tiene un excelente potencial para la cura. Posiblemente la alta tasa de extracciones de glándula submaxilar se relacione con la creencia de que una glándula submaxilar con litiasis es poco funcional. Sin embargo, según estudios realizados por Marchal et $\mathrm{al}^{4,6}$, se ha observado que al tratar la obstrucción se recobra la funcionalidad glándular. En nuestro caso se ha evitado la extirpación glandular en más de la mitad de los pacientes intervenidos $(62,5 \%)$.

En la literatura médica se describe como causa de obstrucción más frecuente la sialolitiasis (el 79\% de las glándulas) con una alta tasa de éxito en su extracción (86\%). Como complicaciones se citan las estenosis posquirúrgicas $(2,5 \%)$ como las más frecuentes, infección posquirúrgica
$(1,6 \%)$, hemorragia posquirúrgica $(0,5 \%)$, ránula traumática y perforación del conducto $(0,7 \%)$ y parestesias del nervio lingual $(0,4 \%)$ como las menos frecuentes ${ }^{5,7,8}$. Un estudio experimental reciente ha utilizado stents arteriales para facilitar el acceso y la protección de los conductos ${ }^{8}$.

La sialoendoscopia es un procedimiento novedoso, con baja morbilidad y pocas molestias, que se puede utilizar para el diagnóstico y el tratamiento de las enfermedades inflamatorias de las glándulas salivales.

\section{BIBLIOGRAFÍA}

1. Marchal F, Dulguerov P. Sialolithiasis management: the state of the art. Otolaryngol Head Neck Surg. 2003;129:951-6.

2. Nahlieli O, Baruchin AM. Sialoendoscopy: three year's experience as a diagnostic and treatment modality. J Oral Maxillofac Surg. 1997;55:912-8.

3. Nahlieli O, Shacham R, Shlesinger M, Eliav E. Juvenile recurrent parotitis: a new method of diagnosis and treatment. Pediatrics. 2004;114:9-12.

4. Marchal F, Kurt AM, Dulquerov P Lehmann W. Rettrograde theory in sialolithiasis formation. Arch Otolaryngol Head Neck Surg. 2001;127:66-8.

5. Nahlieli O, Baruchin A. Long-term experience with endoscopic diagnosis and treatment of salivary gland inflammatory diseases. Laryngoscope. 2000;110: 988-93.

6. Marchal F, Dulguerov P. Sialolithiasis management. Otolaryngol Head Neck Surg. 2003;129:951-6.

7. Nahlieli O, Nakar LH, Nazarian Y, Turner MD. Sialoendoscopy: a new approach to salivary gland obstructive pathology. J Am Dent Assoc. 2006;137: 1394-400.

8. Papadaki M, Kaban L, Kwolek C, Keith D, Troulis M. Arterial stens for acces and protection of the parotid and submandibular ducts during sialoendoscopy. J Oral Maxillofac Surg. 2007;65:1865-8.

9. Capaccio P, Torretta S, Ottavian F, Sambarato G, Pignataro L. Modern management of obstructive salivary diseases. Acta Otorhinolaryngol Ital. 2007;27: 161-72. 